\title{
LA-UR-16-26382
}

Approved for public release; distribution is unlimited.

\section{Title: Expanding the HAWC Observatory}

Author(s): $\quad$ Mori, Johanna

Intended for: Report

Issued: 
Disclaimer:

Los Alamos National Laboratory, an affirmative action/equal opportunity employer, is operated by the Los Alamos National Security, LLC for the National Nuclear Security Administration of the U.S. Department of Energy under contract DE-AC52-06NA25396. By approving this article, the publisher recognizes that the U.S. Government retains nonexclusive, royalty-free license to publish or reproduce the published form of this contribution, or to allow others to do so, for U.S. Government purposes. Los Alamos National Laboratory requests that the publisher identify this article as work performed under the auspices of the U.S. Department of Energy. Los Alamos National Laboratory strongly supports academic freedom and a researcher's right to publish; as an institution, however, the Laboratory does not endorse the viewpoint of a publication or guarantee its technical correctness. 


\title{
Expanding the HAWC Observatory
}

\author{
Johanna Mori
}

August 17th, 2016

\begin{abstract}
The High Altitude Water Cherenkov Gamma-Ray Observatory is expanding its current array of 300 water tanks to include 350 outrigger tanks to increase sensitivity to gamma rays above $10 \mathrm{TeV}$. This involves creating and testing hardware with which to build the new tanks, including photomultiplier tubes, high voltage supply units, and flash analog to digital converters. My responsibilities this summer included preparing, testing and calibrating that equipment.
\end{abstract}

\section{INTRODUCTION}

The High Altitude Water Cherenkov Observatory (HAWC) observes gamma rays, the highest energy part of the electromagnetic spectrum. It currently con- 
sists of 300 water tanks at 4100 meters, or 14000 feet above sea level, on the Sierra Negra mountain in Mexico. HAWC indirectly detects gamma rays with energies of hundreds of $\mathrm{GeV}$ up to about $100 \mathrm{TeV}$ by observing Cherenkov radiation within the water tanks. Gamma rays are blocked by the atmosphere, but that energy can't just disappear. $1 \mathrm{eV}$ of energy is about 1 visible photon; a $1 \mathrm{TeV}$ gamma ray has about the same energy as one trillion visible photons, or one mosquito doing a pushup. The gamma ray interacts with air molecules in the atmosphere to create a cascade of charged particles illustrated in Figure 1, called an air shower, which HAWC detects.

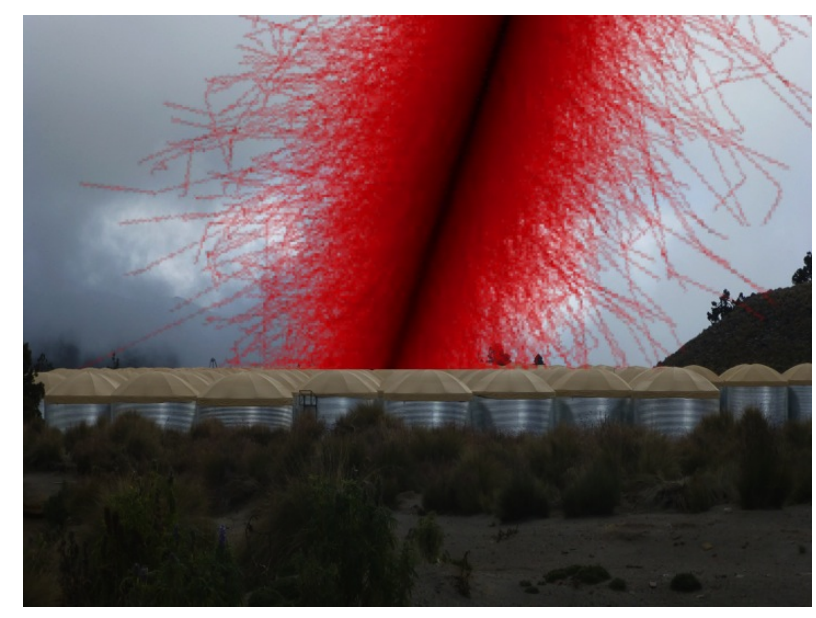

Figure 1: An illustration of an air shower over HAWC

Those charged particles in the shower are moving faster than the speed of light in water, so they create Cherenkov radiation in the tanks. Cherenkov radiation is like the electromagnetic version of a sonic boom; it creates the characteristic glow in nuclear reactors. Four photomultiplier tubes (PMTs) in 
each tank detect the Cherenkov light created by the charged particles speeding through HAWC's tanks, shown in Figure 2. As the gamma ray causes an air shower, the particles spread out like a pancake. By recording when each PMT in each tank saw light, we can see how the shower swept across our array. The PMT timing allows us to recreate the path of the gamma ray, and we can estimate the energy of the gamma ray by extrapolating from the total light seen in all the tanks.

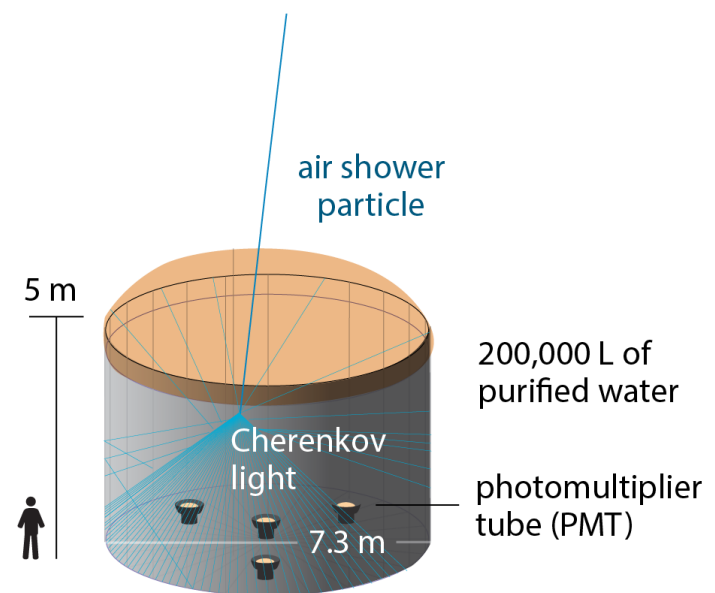

Figure 2: Cherenkov light inside a tank, picked up by the PMTs

HAWC can currently create maps like that seen in Figure 3. However, we would like to increase our sensitivity and improve our resolution and energy estimation by expanding the observatory. To create a larger effective area, we are building an array of 350 new outrigger tanks around the main array of HAWC, as shown in Figure 4. These are smaller tanks, with only one PMT 
instead of four, but they will increase sensitivity to events above $10 \mathrm{TeV}$ by a factor of 2 to 4 , along with improving our spatial resolution and energy estimation.

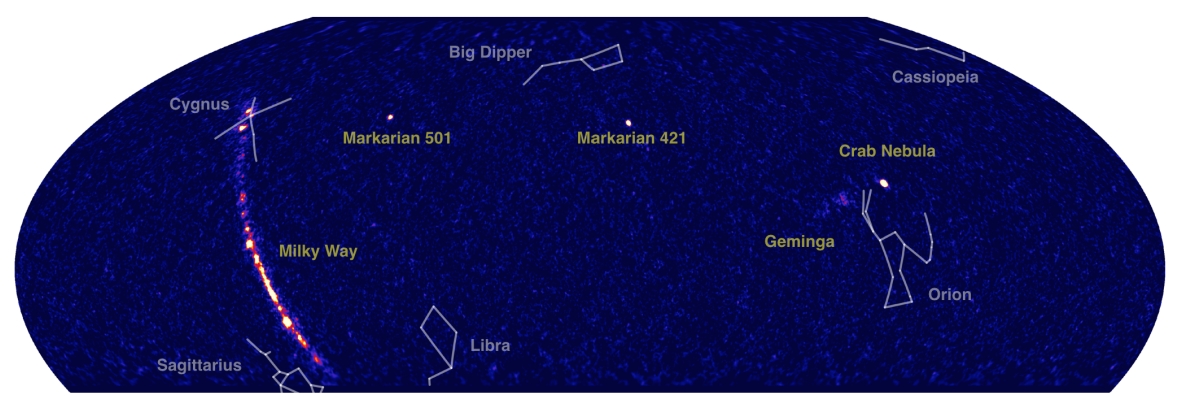

Figure 3: A map of the sky as seen by HAWC.

To expand the observatory, everything has to be tested: water tanks, cables, PMTs, high voltage supply units, and data acquisition hardware. Some equipment, like the water tanks, is being tested in Mexico. LANL is focusing on the PMTs, cables, and understanding the limits of the high voltage supply and data acquisition with flash analog to digital converters (FADCs).

\section{PMTS}

PMTs are one of the most important parts of the observatory, as they are the instruments that actually observe the light. Inside a PMT, a photon hits the 


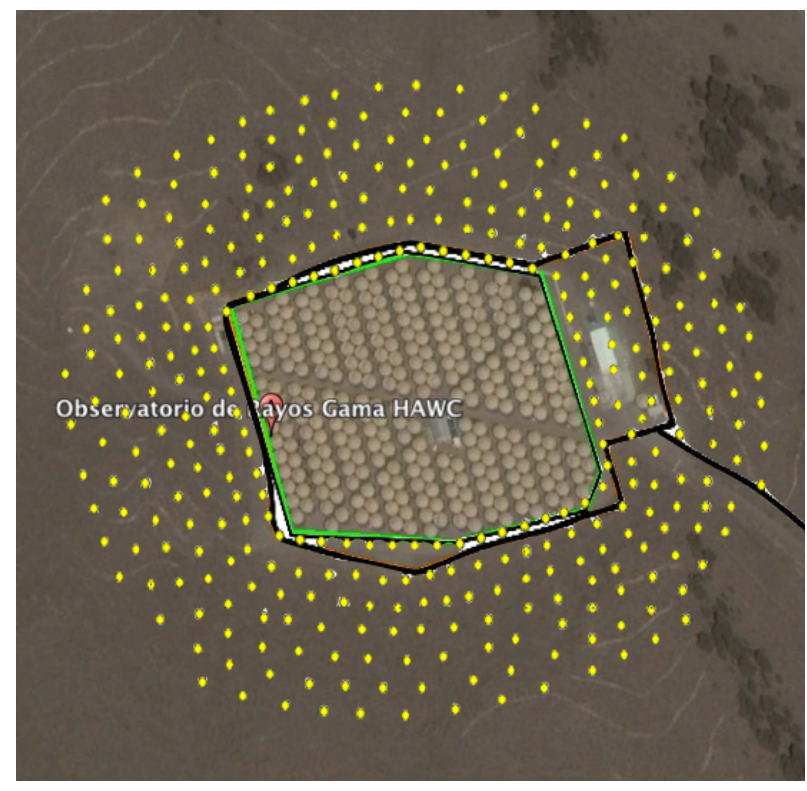

Figure 4: A Google Earth view of HAWC, with proposed locations of the outrigger tanks noted in yellow.

photocathode, knocks off a photoelectron through the photoelectric effect, and an electric field accelerates that photoelectron. The electric field is dependent on the set of resistors in the base of the PMT. When that electron hits the next dynode, it knocks off more electrons, and repeats that process through a chain of dynodes, multiplying the signal into something we can detect.

PMTs are very precise, but each one is just a bit different. As such, we have to test each PMT, so we know how much electrical signal it produces for any given amount of light. This depends on the voltage applied, light levels the PMT sees, and the base design of the PMT. As of now, the PMTs for 
the HAWC outriggers have not been tested. We have only received about a hundred of them, and they still need to have the bases put on them before each of them can be tested en masse. However, we do have a small number of PMTs with the bases, so we can test our other equipment. Several elements go into testing the PMTs: they must have a light source to see, a dark box to keep out unwanted light, a high-voltage supply to power them, and a system to record the data. I have worked on preparing all of those systems this summer.

PMTs are very sensitive instruments; therefore they must be kept in the dark while we're taking data with them to prevent them from being saturated with ambient light. The dark box was built to calibrate the PMTs for the main HAWC array, and has been modified and improved over the years until the wiring labels were inconsistent in different parts of the setup. I cleaned off all the inconsistent labels, and replaced them with a consistent numbering scheme, to make testing the PMTs easier.

The amount of voltage affects PMT performance, by changing the electric field between the dynodes inside the PMT, which changes the PMT gain. Gain is the factor by which one photoelectron gets multiplied by a PMT, usually on the order of $10^{7}$. This matters because we want to maximize the size of small pulses so they are easier to read, but not increase the gain so much that larger pulses saturate the PMT and register as smaller than they actually are. I helped run tests of some PMTs to determine the gain of various bases. We used 
an analog to digital converter (ADC) to measure the charge from the PMTs, then used a fitting program to calculate the gain.

We use filter wheels and a laser to give the PMTs known amounts of light. Our filter wheels have a maximum combined optical density (OD) of 6.36, which means we can reduce the light levels by a factor of up to $10^{6.36}$ or about 2.3 million. The dark box allows us to block out surrounding light and test or calibrate up to 10 PMTs at a time.

Because calibrating the PMTs with various voltages and light levels requires a lot of time, ideally we would be able to set everything up, start a script, and leave the PMTs overnight, and have data in the morning. To

this end, we need to be able to control the FADC, the filter wheels, and the high voltage supply from one computer script. I have spent a lot of time attempting to get all three systems to talk to each other, and we are currently able to communicate with the FADC and making progress on the other systems.

\section{FILTER WHEELS}

Another interesting hurdle was finding out how much light the PMTs are actually seeing. We have a pulsed laser that goes through a series of adjustable 
filters, then through an optical fiber into the dark box. It is possible to use Poisson statistics to determine how many photoelectrons the PMT is seeing when at very small light levels, and then knowing the OD of the filters, that information can be extrapolated out to how much more light the PMTs are seeing at higher light levels. However, that does involve knowing the OD of the filters. Our set included two filter wheels, one of which had OD values of $0,1,2,3,4,5$; and the other which had OD values of $0,0.2,0.4,0.6,0.8,1.0$; at least theoretically. We weren't getting really consistent readings from the PMTs, though, so we decided that we needed to study the filters to determine their actual OD and variation.

As shown in Table 1, the ODs are not quite what they were labeled to be, especially in the set with higher ODs. The ODs found were fairly near the labeled ODs, but the standard deviations were huge. For example, for the filter labeled 3.0, the variation of $+/-0.1428$ means that the measurement could change by a factor of 2. For example, in one set of data, the absolute power of the laser remained steady, but the power fell steadily on filter 3 and steadily gained on filter 4. Possibly, the filter density is not uniform and the filter doesn't return to the same place every time, so we get different values, or there may be some smudge on the filters, but the result is that our filter wheels are less accurate than desirable. 
Table 1 was created using a 4.5 milliWatt continuous beam laser and a photodiode. The beam passed through the filters and then a lens to focus the light into a fiber and then onto the photodiode.

We used the continuous laser because the power output of the pulsed laser was too low to be read by the photodiode. Through experiment and perusal of the manuals for the radiometer, we found out that our pulsed laser is not compatible with the equipment, because the peak power is too high and the average power is too low. The continuous laser's power output is lower than the maximum power of the photodiode, and because it is continuous, the average power is not too low. However, the uncertainties are rather large so we don't always know exactly how many photoelectrons we're giving to the PMTs.

\section{Cables}

Once we have signals from the PMTs, we have to record them in some meaningful way. The main array of HAWC can only record the total time in which the electrical signal from the PMT was over a certain threshold. The FADCs that the outriggers will use actually records the shape of the pulse. FADCs allow the total signal to be found by integrating, but more detailed information can also be recorded. 
Unfortunately, the signals from the PMTs cannot be instantly beamed back to the FADCs in the outbuildings for the computers. They must travel through high-voltage cables. To build the outrigger array, we need to get all of the signals from the PMTs in the outrigger tanks to the computers in the nodes. Our reconstruction software relies on the time at which the PMT signals come into the computers to trace the gamma ray back to the source, so the times must not be skewed by cables of different lengths delaying some signals by tens of nanoseconds relative to other signals. Therefore, all the high voltage cables must be the same length. Luckily, the computer system needs groups of 24 to be identical, and can be calibrated to compensate for some variation between the batches.

To measure the length of the cables, I used a pulse generator and an oscilloscope shown in Figure 5. The pulse generator had a $\mathrm{T}$ on it, one side of which went to the cable, the other to the oscilloscope. The pulse would go out from the generator to the oscilloscope on one side and the cable on the other. The scope would trigger on the first pulse, then record the reflected pulse off the open end of the cable. Then I could measure the time between the trigger and a consistent spot on the reflected pulse, and convert that to a length with this equation:

$$
2 t v-C,
$$




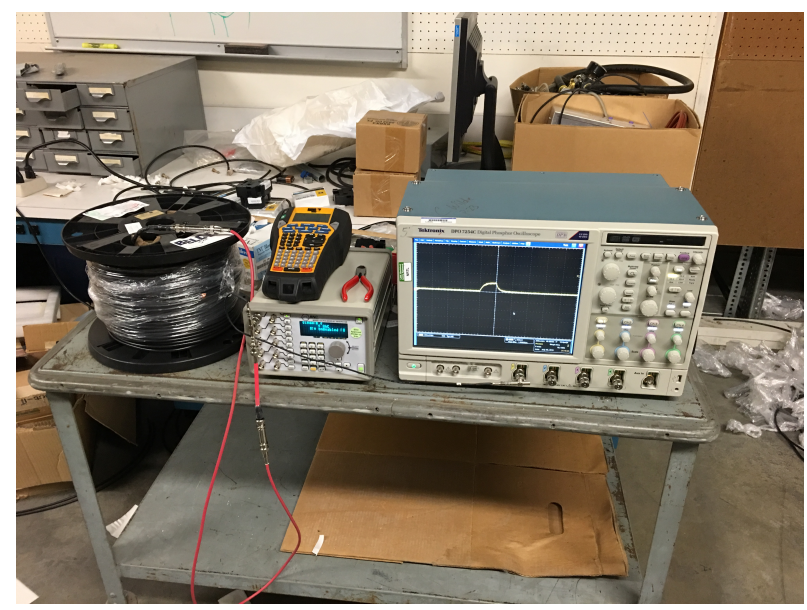

Figure 5: Setup for measuring cables, including a cable spool, pulse generator, and oscilloscope.

where $\mathrm{t}$ is the time between pulse and reflection in nanoseconds; $\mathrm{v}$ is $0.66 \mathrm{ft} / \mathrm{ns}$, the speed of electrical signals in copper wire; multiplied by 2 because the signal has to travel the cable length twice; and $\mathrm{C}$ is $10.33 \mathrm{ft}$, the correction for our adapter cable.

I measured 24 cables, took the shortest and subtracted that length from the length of all of the cables, and then cut that amount off of each cable, so that they were all a uniform length. I was expecting the cables to have some variation, but I was shocked how many cables labeled 500 feet were actually over 700 feet long. After I cut the cable, I would measure it again and record the new length. Some statistics on the cables are shown in Table 2. 
Even after cutting, the batches still have ranges on the order of feet. It was very difficult to achieve better results than this, because I was measuring to a specific point on the reflected pulse by eye, and small variations in the pulse shape could lead to measurement uncertainties around six inches. In addition, while our computer systems need the signals from the PMTs to arrive within a certain time window to trigger the recording software, variations in cable length up to a few nanoseconds can and will be calibrated out of the system.

\section{Conclusion}

At the moment, ten outriggers are under construction at the site in Mexico. All 350 should be finished and contributing data within a year. This should increase our sensitivity and will hopefully contribute to many new discoveries.

Overall, I feel like I have made significant contributions to the expansion of the HAWC observatory. I learned a lot about the practical aspects of lab work, as well as the theoretical side of indirectly observing gamma rays. 


\section{Acknowledgments}

I would like to thank and acknowledge the Department of Energy for funding the SULI program that allowed me to be here; Brenda Dingus, my fantastic mentor; Andrea Albert, Kelly Malone, and Izzy Brewer for being great officemates; and Pat Harding, Patrick Younk, Scott Delay, and Gerd Kunde for all their help throughout the summer. 
Table 1: Optical Densities of Filter Wheels

\begin{tabular}{|ccc|}
\hline \hline Labeled OD & Measured OD & Standard Deviation \\
\hline 0.2 & 0.1679 & 0.0215 \\
0.4 & 0.4304 & 0.0412 \\
0.6 & 0.6346 & 0.0527 \\
0.8 & 0.7937 & 0.0696 \\
1.0 & 1.0436 & 0.0816 \\
1.0 & 1.0251 & 0.0314 \\
2.0 & 2.0478 & 0.0658 \\
3.0 & 2.9165 & 0.1428 \\
4.0 & 3.9983 & 0.1331 \\
5.0 & 5.3181 & 0.0132 \\
\hline
\end{tabular}


Table 2: Average, range, and standard deviation of each batch of cables both before and after cutting.

\begin{tabular}{|l|ccc|ccc|}
\hline \hline Batch Numbers & Average & Range & St. Dev. & Average & Range & St. Dev. \\
\hline $1-24$ & 503.47 & 41.02 & 8.11 & 500.25 & 0.91 & 0.24 \\
$25-48$ & 523.60 & 133.93 & 38.90 & 500.31 & 2.54 & 0.52 \\
$49-72$ & 501.54 & 17.49 & 3.24 & 498.69 & 1.24 & 0.32 \\
$73-96$ & 501.94 & 5.47 & 1.28 & 498.49 & 1.16 & 0.29 \\
$96-120$ & 502.54 & 6.47 & 1.37 & 498.53 & 1.53 & 0.37 \\
$121-144$ & 500.64 & 6.05 & 1.40 & 497.02 & 0.99 & 0.28 \\
$145-168$ & 501.76 & 5.97 & 1.33 & 497.45 & 0.95 & 0.25 \\
$169-192$ & 501.71 & 4.21 & 1.217 & 499.63 & 2.90 & 0.58 \\
$193-216$ & 508.99 & 176.40 & 35.48 & 498.79 & 0.91 & 0.18 \\
$217-240$ & 510.71 & 248.58 & 50.59 & 499.27 & 1.24 & 0.31 \\
$241-264$ & 501.54 & 17.55 & 3.48 & 498.92 & 1.40 & 0.32 \\
$265-288$ & 500.3 & 4.39 & 1.05 & 498.88 & 1.06 & 0.32 \\
$289-312$ & 501.04 & 14.01 & 2.58 & 495.79 & 0.72 & 0.22 \\
\hline
\end{tabular}

